\title{
Synthesis, Characterization and Conductivity Properties of Polystyrene/Polyacrylonitrile/Polyindole Ternary Composites
}

\author{
C. Soykan* and O.C. Candoğan
}

Department of Materials Science and Nanotechnology, Faculty of Engineering, University of Uşak, 64200Uşak, Turkey

\begin{abstract}
In this investigation, polystyrene/polyacrylonitrile/polyindole (PSt/PAN/PIN) ternary composites with various amounts of PSt, PAN and PIN were synthesized using $\mathrm{FeCl}_{3}$ as an oxidant agent by chemical polymerization technique. The formation of ternary composites was assisted by Fourier transform infrared spectroscopy. Morphological studies demonstrated all composite have a smooth surface. The results of thermal gravimetric analysis indicate that incorporation of PIN in composites advances the thermal stability. X-ray diffraction analysis show that the amorphous nature of PIN and its ternary composites. The conductivities of PIN and the PSt/PAN/PIN composites were investigated with a four-probe technique. The conductivity of PIN was found to be $5.0 \times 10^{-3} \mathrm{~S} \mathrm{~cm}-1$, also the conductivities of the PSt/PAN/PIN composites were determined to $3.5 \times 10^{-4}, 7.4 \times 10^{-4}, 1.3 \times 10^{-3}, 2.2 \times 10^{-3}$ and $2.8 \times 10^{-3} \mathrm{~S} \mathrm{~cm}^{-1}$ respectively, with $9,27,45,63$ and $81 \mathrm{wt} \%$ of PIN.
\end{abstract}

Keywords: Chemical Synthesis, Ternary Composites, Conducting Polymers, Thermogravimetry.

\section{INTRODUCTION}

As a material class, conductive polymers have a few characteristics including easy synthesis, macromolecular character, electrical handling properties, and environmentally friendly. Conductive polymers with these properties were used in further applications such as electrochromic tools, sensors, rechargeable batteries and anti-corrosion primer layers [1-7]. In conductive polymers, polyindole (PIN) is specially promising for industrial applications due to its easy synthesis, high redox activity and higher conductivity than many other conductive polymers [8]. PIN which is $\mathrm{N}$-including heteroaromatic organic compound has very interesting features. It is a polymer derived from the polymerization of indole; an aromatic bicyclic structured compound consisting of a sixmembered benzene ring fused to a five-membered nitrogen-containing pyrrole ring [9]. Also, it can be obtained by chemical oxidation of indole in in aqueous media and different organic solvents. In the light of the information collected from polyprol studies, many information and expressions about the structure of the PIN and its polymerization mechanism have been included in the literature [10-13]. The difference of chemical synthesis is that it preferences mass making at a appropriate cost. Besides, chemically synthesized PIN, compared to the traditional conductive polymers has been comparatively less investigated. Polystyrene (PSt) and polyacrylonitrile (PAN) were chosen as an

*Address correspondence to this author at the Department of Materials Science and Nanotechnology, Faculty of Engineering, University of Uşak, 64200-Uşak, Turkey; Tel: +90 276 2212136/2701; Fax: +90 276 2212122; E-mail: cengizsoykan@usak.edu.tr insulating matrix because of its unique properties [14]. They are important commercial plastic and is attractive candidates as components for synthesis of composites. PSt very affordable offers lightness and durability, high thermal stability and exhibits mechanical strength [15]. Hall effect measurements are important for semiconductor material characterization because of the information which may be obtained, that contains mobility, carrier density and temperature-dependent conductivity. These parameters are very important in the polymer and composite synthesis, and device design. PIN is used as a conducting matrix in the ternary composites because of its high conductive properties [16].

To the best our knowledge, there are no reports in literatüre on the synthesis of PSt/PAN/PIN ternary composites. Besides, with synthesizing conductive PSt/PAN/PIN composites not only literature will have been added innovation and productiveness but also some features of PIN will have been advanced with modifying. With composite synthesis, the thermal stability of PAN and PSt will have been significantly progressed.

\section{EXPERIMENTAL DATAILS}

\subsection{Reagents}

Indole ( $\geq 99 \%$ pure) was supplied from Merck, Germany. Acrylonitrile (AN-Sigma-Aldrich), Styrene (St-Sigma Aldrich) were used in polymerizations after they were expelled with a dilute $\mathrm{KOH}$-base solution in the hydroquinone inhibitor. The benzoylperoxide (BPO) used to initiate free radical polymerization was 
dissolved in chloroform and recrystallized by precipitation in methanol. Argon gas as inert gas in polymerization processes; 1,4-dioxane was used as the solvent and ethanol was used as the precipitant. 1,4dioxane, chloroform, ethanol (Merck), were analytical grade commercial products and used as received.

\subsection{Methods of Characterization}

Fourier transform infrared (FT-IR) spectra of the samples were recorded on Perkin Elmer Spectrum Two (UATR) IR Spectrometer in the range of $400-4000$ $\mathrm{cm}^{-1}$. TGA analysis was carried out using a Hitachi 7000 TGA/DTA model thermogravimetry device in the accompanie of nitrogen atmosphere up to $1000^{\circ} \mathrm{C}$, at a heating rate of $10^{\circ} \mathrm{C} / \mathrm{min}$. The surface morphologies of the synthesized materials was investigated using a Zeis EVO LS10 model scanning electron microscope (SEM) operated at $15 \mathrm{kV}$. XRD study of the nanocomposites was performed on a Rigaku Dmax2200 XRD Model X powder diffractometer, using CoKa radiation whose wavelength was $0,178901 \mathrm{~nm}$, and a Ni filter was used. Conductivities of the polymer and ternary composites were measured by a PC Card 1716 PCLD 8710 model computer, a four probe instrument, on pressed pellets at room temperature.

\subsection{Homopolymerization of Styrene (PSt) and Acrylonitrile (PAN)}

8 grams of St and AN monomers were taken into two separate polymerization tubes. It was dissolved in 1,4-dioxane and passed through argon gas with $1 \%$ benzoylperoxide (BPO) initiator to provide polymerization at $70{ }^{\circ} \mathrm{C}$. The resulting homopolymers were purified by precipitation the three times with ethyl alcohol to remove impurities. It was first dried in an atmosphere of air, then at $40{ }^{\circ} \mathrm{C}$, and stored for analysis in a flask.

\subsection{Synthesis of PSt/PAN/PIN Ternary Composites}

PSt/PAN/PIN ternary composites of $9,27,45,63$, and 81 wt \% of PIN were prepared using $\mathrm{FeCl}_{3}$ as an oxidant and by a chemical oxidative polymerization technique at $25^{\circ} \mathrm{C}$. In the synthesis of all composites, the same molar ratio of oxidant to monomer was receipted as 2:1. Firstly, $\mathrm{FeCl}_{3}$ was dissolved in chloroform in a three-necked flask. Then 1.8, 1.4, 1.0, 0.6 , and $0.2 \mathrm{~g}$ PSt and PAN were dissolved in chloroform and added into the stirred oxidant solution, respectively. After 15 minute of stirring, the indole monomer (dispersed in chloroform) was added dropwise into this solution under the nitrogen gas atmosphere. The reaction temperature was kept steady at $25^{\circ} \mathrm{C}$. After 30 hour the precipitate was filtered and washed with chloroform and distilled hot water and dried in a vacuum oven at $45^{\circ} \mathrm{C}$ for 24 hour. Finally, the composition of the PSt/PAN/PIN ternary composite was calculated gravimetrically from the total mass of the composite. As a result, PSt/PAN/PIN ternary composites containing PIN at different quantity were obtained. Synthesis schema of PStPAN/PIN composite is given in Figure 1:

\section{RESULTS AND DISCUSSION}

\subsection{FTIR Analysis}

Figure 2 shows FTIR spectra of PSt, PAN, and PSt/PAN/PIN ternary composites.

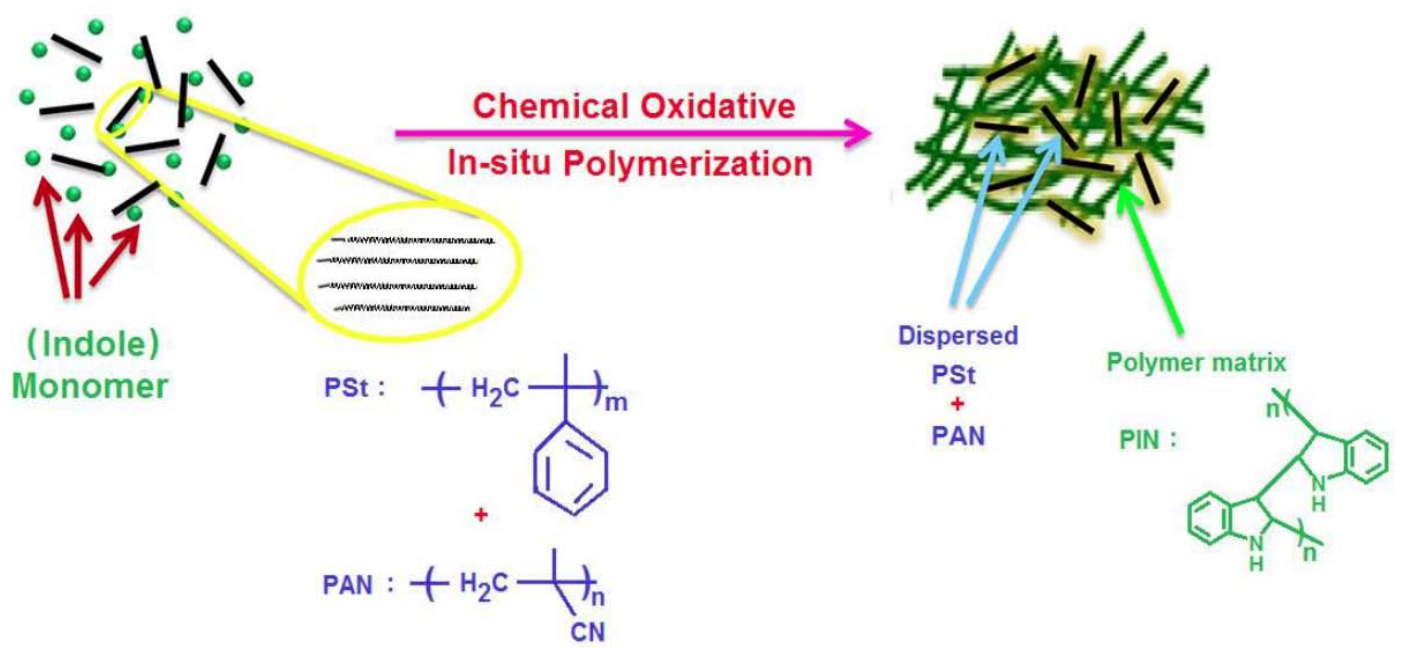

Figure 1: Synthesis schema of the PSt/PAN/PIN ternary composites. 


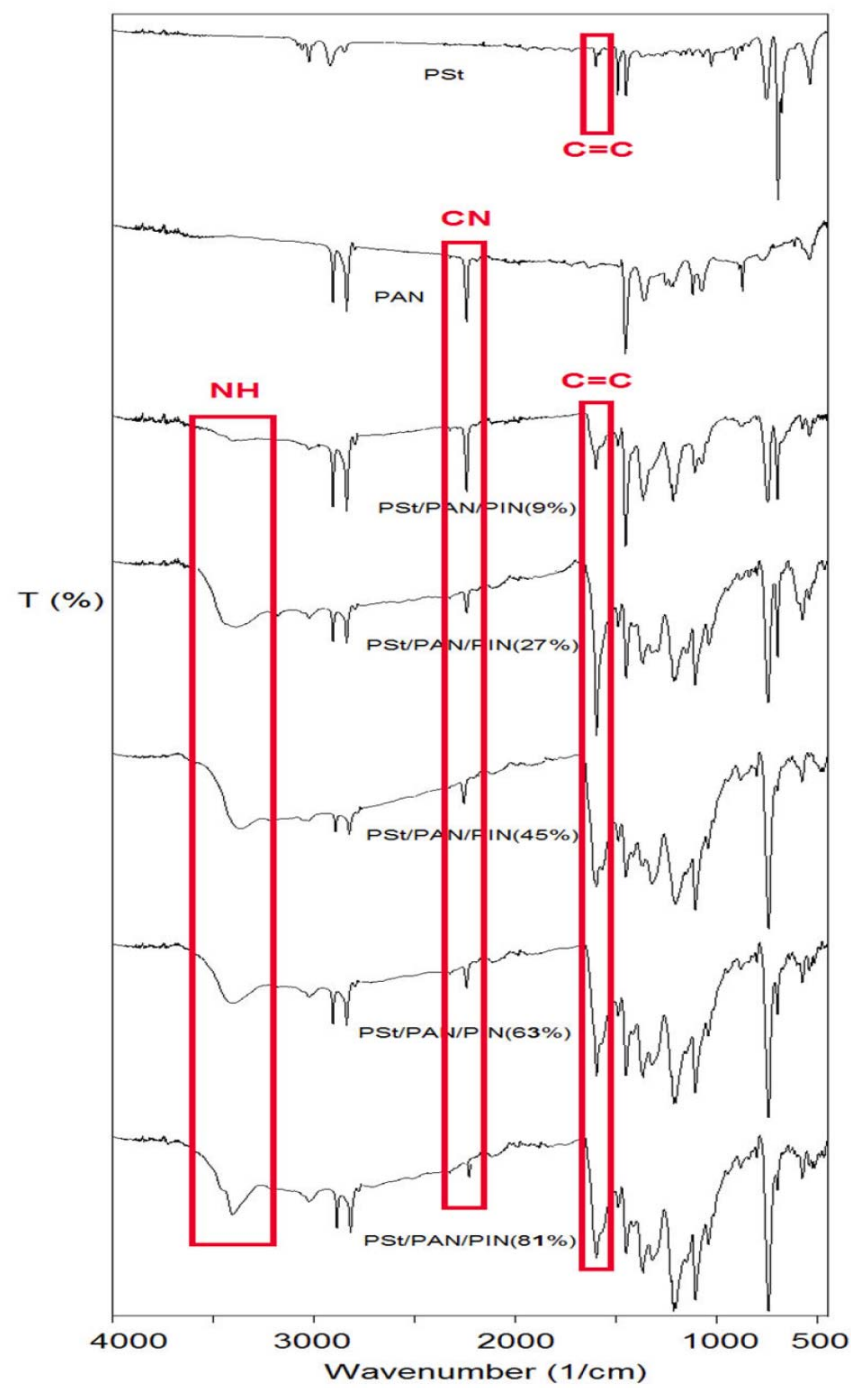

Figure 2: FTIR spectra of the PSt, PAN, and PSt/PAN/PIN ternary composites.

The pure PSt exhibits several characteristic absorption bands at 3030-2900 and at 1600-1450 $\mathrm{cm}^{-1}$, which can be assigned to the $\mathrm{C}-\mathrm{H}$ stretching vibration and the $\mathrm{C}=\mathrm{C}$ stretching vibration, respectively [17]. The absorption infrared spectrum of PAN shows the tensile vibration of the characteristic $\mathrm{CN}$ group at $2250 \mathrm{~cm}^{-1}$ and the $\mathrm{C}-\mathrm{H}$ aliphatic tension is $2900 \mathrm{~cm}^{-1}$. In the FT-IR spectrum of PSt/PAN/PINs, the bands at 3390,1500 , and $1405 \mathrm{~cm}^{-1}$ specify the presence of the $\mathrm{N}-\mathrm{H}$ stretching in the PIN unit. The band at $1580 \mathrm{~cm}^{-1}$ could be pointed to the vibration of the $\mathrm{C}=\mathrm{C}$ aromatic bonds typical of indoles. One band at $745 \mathrm{~cm}^{-1}$ show $\mathrm{C}-\mathrm{H}$ vibration of PIN. This band indicates the presence of a hydrogen bond at the end of the chain. Also, the band at $1500 \mathrm{~cm}^{-1}$ can be related to the $\mathrm{N}-\mathrm{H}$ stretch and $\mathrm{C}=\mathrm{C}$ deformation. The bands at 1455 and 1205 $\mathrm{cm}^{-1}$ are pointed to the benzene and aromatic rings of PIN molecule structure.

\subsection{Powder X-Ray Diffraction}

XRD powder patterns of PSt, PAN, PIN, and PSt/PAN/PIN ternary composites with different weight percentages $(9,45$, and 81$)$ are shown in Figure 3. $\mathrm{XRD}$ analysis was conducted to evaluate $\mathrm{X}$-ray curves of the composites by comparing to each other. The XRD diagrams exhibited broad diffraction peaks, suggesting that all of the polymers were amorphous. XRD pattern of PIN shows two broad diffraction peak at approximately $2 \theta=20^{\circ}$ and $2 \theta=26^{\circ}$, which implies the existence of amorphous polyindole [18-22]. As far as

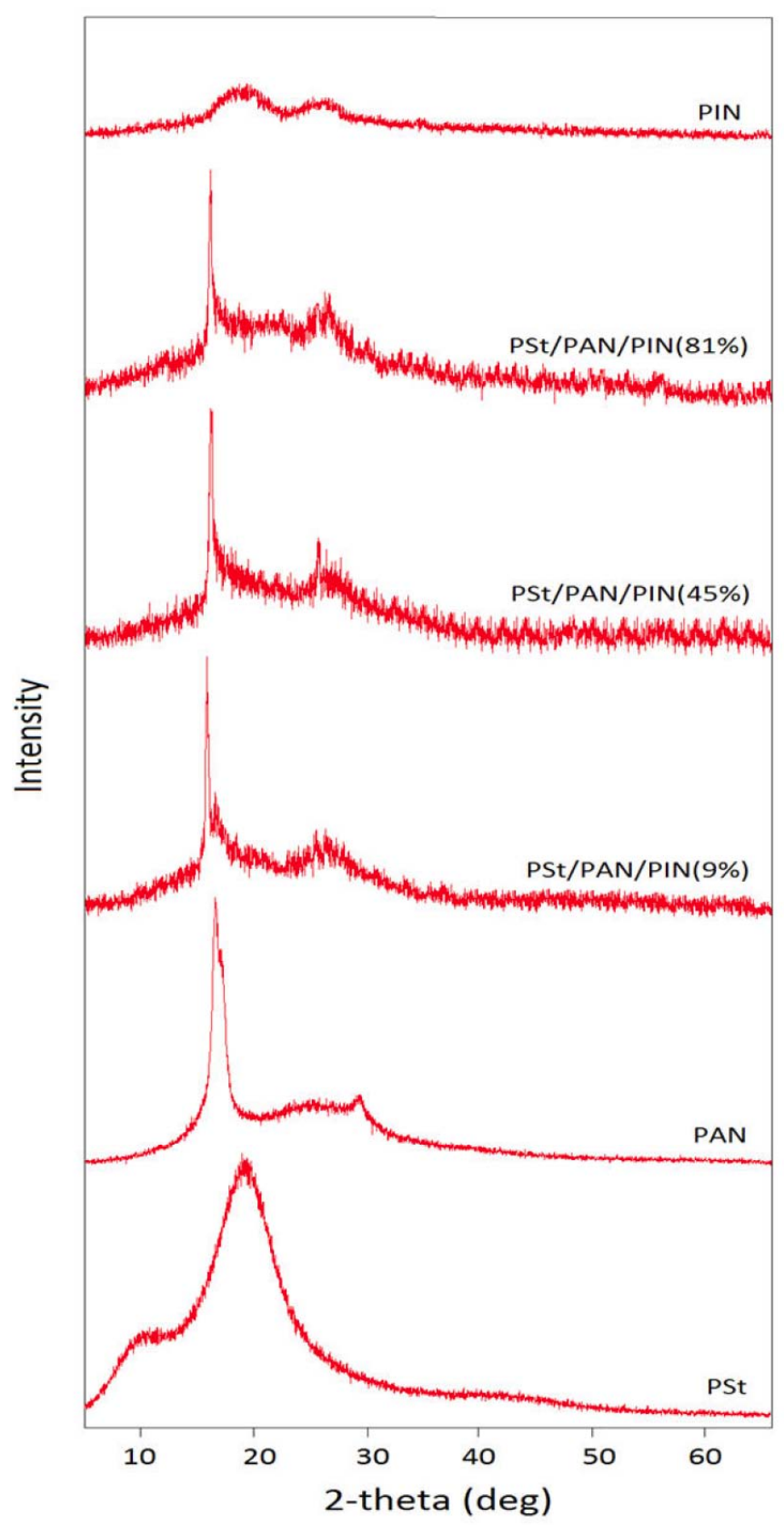

Figure 3: XRD pattern of the PSt, PAN, PIN and PSt/PAN/PIN ternary composites. 

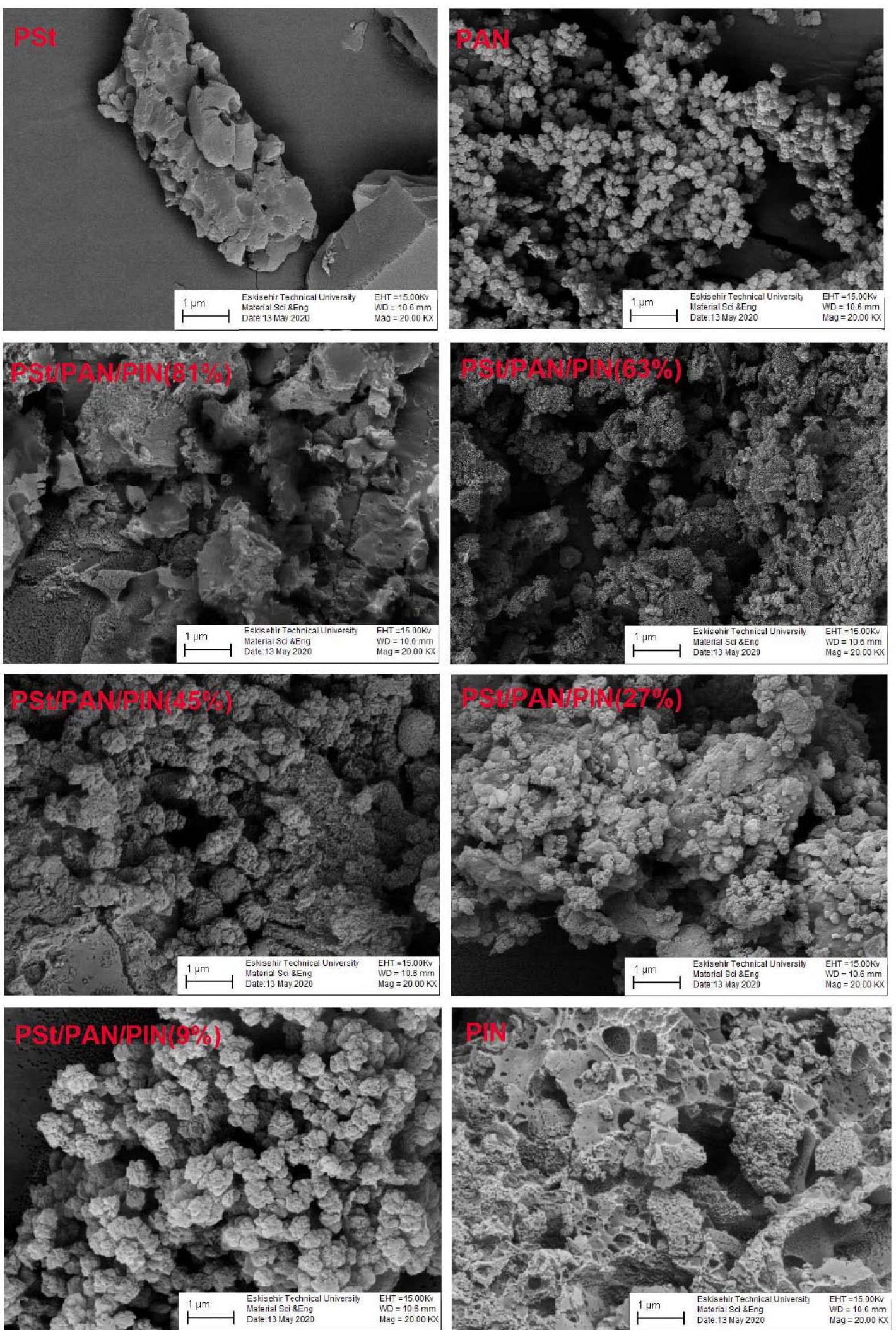

Figure 4: SEM images of the PSt, PAN, PIN and PSt/PAN/PIN ternary composites.

we known that PSt and PAN are amorphous and transparent polymers. The broad amorphous diffraction peak observed at around $2 \theta=21^{\circ}$ in the XRD curve of
PS. The broad amorphous diffraction peaks were indicated at around $2 \theta=15-30^{\circ}$ and $20^{\circ}$ in the XRD curves of PSt and PAN, respectively [23]. PSt/PAN/PIN 
ternary composites show an amorphous structure, as a consequence of the amorphous nature of PSt, PAN, and PIN. As a result, X-ray curves shifted to larger angles and amorphousness was increased with increasing PIN content in the composite.

\subsection{Morphology Analysis}

As known that the properties of materials substantially related to their morphological structure integrity. The distribution of the components is highly important for composites. SEM images of chemically synthesized PSt, PAN, PIN and PSt/PAN/PIN composites including $9,27,45,63$, and $81 \mathrm{wt} \%$ of PIN, have been seen in Figure 4. The SEM image of PIN shows a sponge-like, granular structure, porous, which is easy going with literature [24]. The SEM image of PSt indicates unitary, layered and veined structure. The SEM micrograph of PAN shows cauly-flower structure. When the morphologies of PSt, PAN and the composites were compared, it was conducted that the composites had quite privilege morphologies with PSt and PAN. With the growing PIN quantity in the composites, the surface properties of the composites point out the similar morphology of PIN. It is well known that the electrical conductivity connected with both number of charge carriers and the mobility that can be related to chemical composition and morphology of polymers or composites [25].

As seen by the SEM micrographs, PIN presents a normal granular structure while the PSt and PAN specifies unitary, layered and veined morphology. By this occasion, the surface morphology of a polymer or composite affectes its conductivity [26, 27]. Besides, the conductivity values have been given PIN weight fraction in Table 1. As may be realized from the table the conductivities of the PSt/PAN/PIN composites was

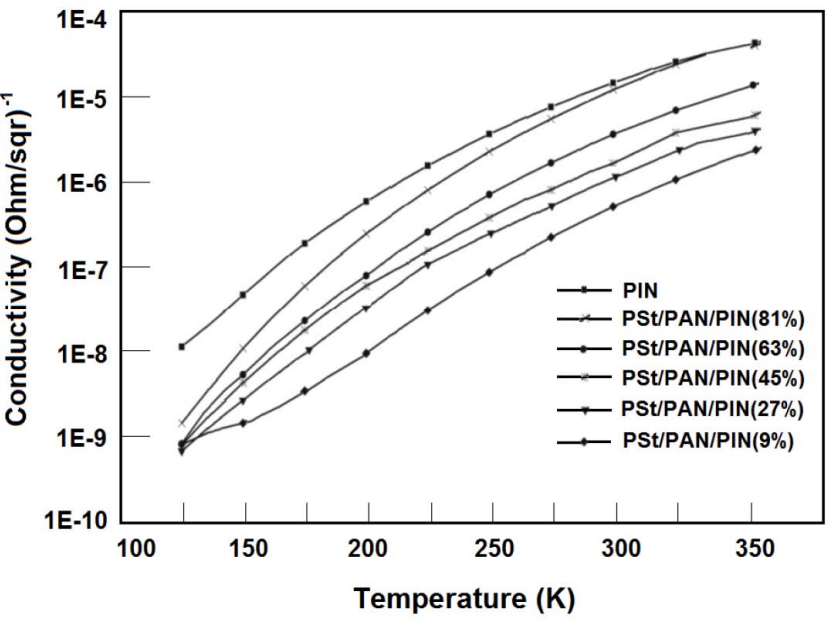

Figure 5: Temperature dependence of the conductivity for PIN and PSt/PAN/PIN with 81, 63, 45, 27 and 9 wt \% of PIN.

increased by increasing the quantity of PIN. Briefly, with increasing the PIN content in composite, electrical conductivity increases regularly, this depends on the contact distance between the conductive particles or the conductive path.

\subsection{Electrically Conductivity}

Electrically conductive PSt/PAN/PIN composites were synthesized and effect of temperature on the electrical conductivity was investigated. The temperature dependent conductivities for PIN and PSt/PAN/PIN composites are shown in Figure $\mathbf{5}$.

The electrical conductivity may be expressed as the following equation, $\sigma=n q \mu$ equation. Where $n$ is the number of electrons per unit volume, $q$ the magnitude of the electrical charge on an electron, and $\mu$ is pointed out the electron mobility. As a result, the electrical conductivity is proportional to both the number of electrons and the electron mobility. As shown in Table 1 , the conductivity increases with the quantity of

Table 1: Polymerization Yield and Conductivity of the Powder Composites and Polymers

\begin{tabular}{|c|c|c|}
\hline Composite, Polymer & $\begin{array}{c}\text { Polymerization Yield of PIN in Ternary } \\
\text { Composite (wt \%) }\end{array}$ & \begin{tabular}{c} 
Conductivity, $\boldsymbol{\sigma}\left(\mathbf{S ~ c m}{ }^{-1}\right)$ \\
\hline PSt/PAN/PIN(9\%)
\end{tabular} 92 \\
\hline \hline PSt/PAN/PIN(27\%) & 94 & $7.4 \times 10^{-4}$ \\
\hline PSt/PAN/PIN(45\%) & 93 & $1.3 \times 10^{-3}$ \\
\hline PSt/PAN/PIN(63\%) & 93 & $2.2 \times 10^{-3}$ \\
\hline PSt/PAN/PIN(81\%) & 92 & $2.8 \times 10^{-3}$ \\
\hline PIN & 94 & $5.0 \times 10^{-3}$ \\
\hline PSt & 95 & - \\
\hline PAN & 95 & - \\
\hline
\end{tabular}


conductive PIN in the composites and temperature. By increasing quantity of PIN, the number of electroactive centers increases, and therefore the conductivity increases [19,24]. The increase in temperature causes an increase segmental mobility and in free volume. Increasing temperature tends to increase the number of charge carriers and electronic movement, consequently it ends up with higher electrical conductivity $[18,24]$. The increase of conductivity with increasing temperature specifies classic semiconductor behavior, unlike decreasing conductivity is a typical of metals.

\subsection{Thermo-Physical Property of Polymers and Composites}

Thermal stability of PSt, PAN and PSt/PAN/PIN composites including various quantity of PIN were performed by TGA and results are seen in Figure 6.

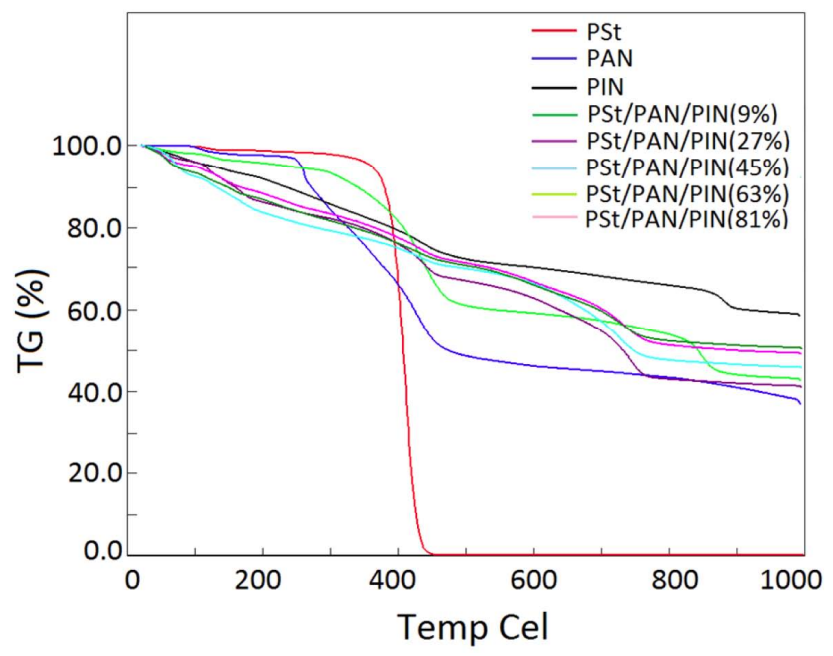

Figure 6: TGA curves of the PSt, PAN, PIN and PSt/PAN/PIN ternary composites.

In different decomposition temperatures at \%weight loss and residue $\%$ at $1000{ }^{\circ} \mathrm{C}$ calculated from these curves are given in Table 2. As can be seen in Figure 6. PSt showed a one-stage weight loss, PAN and PSt/PAN/PIN composites showed a two-stage weight loss. The weight loss in the first stage due to the loss of moisture, gas outlets such as carbon monoxide and dopant. The second stage may be pointed out to the complete degradation and decomposition of the polymer $[28,29]$. The thermal degradation of PSt arises between and $382-420{ }^{\circ} \mathrm{C}(100 \%$ weight loss) [30]. Figure 6 shows the thermograms of PSt/PAN/PIN composites including different quantity of PIN. Composites show a two-stage decomposition pattern. From the TGA curves the PSt/PAN/PIN composite which included $9 \%$ of PIN has the highest decomposition temperature with in all the composites. According to the result, the thermal stability of composites was raised with increasing PAN and PIN quantity.

\section{CONCLUSIONS}

In the present study, PSt/PAN/PIN ternary conductive composites were successfully synthesized by chemical polymerization method. It was modified the spectroscopic, thermal, morphological, and conductivity properties of these ternary conducting composites. After then, the conductivity PSt/PAN/PIN ternary composites were measured using the four prob technique. This parameter was discussed as a function of PIN concentration and temperature. As we understand from the results we have achieved from the discussion, the conductivity increased with increasing temperature. We obtained from SEM and X-ray results that the structures of the PSt, PAN and PSt/PAN/PIN composites had amorphous regions. The PSt/PAN/PIN composites show advanced thermal stability compared to the pure PSt and PAN, especially in the temperature range $400-800{ }^{\circ} \mathrm{C}$. Because of these features, PSt/PAN/PIN composites may be used in areas such

Table 2: TGA Results of the Samples

\begin{tabular}{|c|c|c|c|c|}
\hline Composite, Polymer & $\begin{array}{l}\text { Temperature of } 50 \% \\
\text { weight loss }\left({ }^{\circ} \mathrm{C}\right)\end{array}$ & $\begin{array}{l}\% \text { Weight loss } \\
\text { (at } 400{ }^{\circ} \mathrm{C} \text { ) }\end{array}$ & $\begin{array}{l}\% \text { Weight loss } \\
\text { (at } 600^{\circ} \mathrm{C} \text { ) }\end{array}$ & $\begin{array}{l}\% \text { Residue } \\
\text { (at } 1000{ }^{\circ} \mathrm{C} \text { ) }\end{array}$ \\
\hline PSt/PAN/PIN(9\%) & 844 & 17 & 38 & 43 \\
\hline PSt/PAN/PIN(27\%) & 780 & 23 & 34 & 41 \\
\hline PSt/PAN/PIN(45\%) & 830 & 22 & 30 & 50 \\
\hline PSt/PAN/PIN(63\%) & 786 & 25 & 29 & 45 \\
\hline PSt/PAN/PIN(81\%) & 820 & 22 & 29 & 52 \\
\hline PIN [31] & - & 12 & 17 & 68 \\
\hline PSt & 422 & 34 & 0 & 0 \\
\hline PAN & 490 & 33 & 52 & 38 \\
\hline
\end{tabular}


as light-emitting diode (LED), field-effect transistor (FET) materials, antistatic materials etc.

\section{CONFLICT OF INTEREST}

The authors declare that they have no conflict of interest.

\section{REFERENCES}

[1] Pang Y, Li X, Ding H, Shi G, Jin L. Electropolymerization of High Quality Electrochromic Poly(3-Alkyl-Thiophene)s Via a Rroom Temperature İonic Liquid. Electrochimica Acta 2007; 52 (20): 6172-6177.

https://doi.org/10.1016/j.electacta.2007.04.015

[2] Navarchian AH, Hasanzadeh Z, Joulazadeh M. Effect of Polymerization Conditions on Reaction Yield, Conductivity, and Ammonia Sensing of Polyaniline. Advances in Polymer Technology 2013; 32: 21356.

https://doi.org/10.1002/adv.21356

[3] Baibarac M, Lira-Cantứ M, Oró Sol J, Baltog I, Casan-Pastor $\mathrm{N}$, Gomez-Romero P. Poly(N-Vinyl Carbazole) and Carbon Nanotubes Based Composites and Their Application to Rechargeable Lithium Batteries. Composites Science and Technology 2007; 67 (11-12): 2556-2563. https://doi.org/10.1016/j.compscitech.2006.12.008

[4] Castagno KRL, Dalmoro V, Mauler RS, Azambuja DS. Characterization and Corrosion Protection Properties of Polypyrrole/Montmorillonite Electropolymerized onto Aaluminium Alloy 1100. Journal of Polymer Research 2010; 17 (5): 647-655.

https://doi.org/10.1007/s10965-009-9353-0

[5] Wu H, Fahy WP, Kim S, Kim H, Zhao N, Pilato L, Kafi A, Bateman S, Koo JH. Recent Developments in Polymers/ Polymer Nanocomposites for Additive Manufacturing. Progress in Materials Science 2020; 111: 100638. https://doi.org/10.1016/j.pmatsci.2020.100638

[6] Gueye MN, Carella A, Vincent JF, Demadrille R, Simonato JP. Progress in Understanding Structure and Transport Properties of PEDOT-Based Materials: A Critical Review. Progress in Materials Science 2020; 108: 100616. https://doi.org/10.1016/j.pmatsci.2019.100616

[7] Umoren SA, Solomon MM. Protective Polymeric Films for Industrial Substrates: A Critical Review on Past and Recent Applications with Conducting Polymers and Polymer Composites/Nanocomposites. Progress in Materials Science 2019; 104: 380-450. https://doi.org/10.1016/j.pmatsci.2019.04.002

[8] Çoğal S, Kiristi M, Ocakoğlu K, Öksüz L, Öksüz AU. Electrochromic Properties of Electrochemically Synthesized Porphyrin/3-Substituted Polythiophene Copolymers. Materials Science in Semiconductor Processing 2015; 31: 551-560.

https://doi.org/10.1016/j.mssp.2014.12.036

[9] Matsui H, Hasegawa T, Tokura Y, Hiraoka M, Yamada T. Polaron Motional Narrowing of Electron Spin Resonance in Organic Field-Effect Transistors. Physical Review Letters 2008; 100(12) 126601.

https://doi.org/10.1103/PhysRevLett.100.126601

[10] Majumdar HS, Botta C, Bolognesi A, Pal AJ, Memory Applications of a Thiophene-Based Conjugated Polymer by Photoluminescence Measurements. Synthetic Metals 2005; 148: $175-178$.

https://doi.org/10.1016/j.synthmet.2004.09.021

[11] Saraji M, Bagheri A. Electropolymerization of Indole and Sstudy of Electrochemical Behavior of the Polymer in Aqueous Solutions. Synthetic Metals 1998; 98(1): 57-63. https://doi.org/10.1016/S0379-6779(98)00151-9
Talbi H, Monard G, Loos M, Billaud D. Theoretical Investigation of the Monomer Reactivity in Polyindole Derivatives. Synthetic Metals 1999; 101(1-3): 115-116. https://doi.org/10.1016/S0379-6779(98)00080-0

[13] Billaud D, Humbert B, Thevenot L, Thomas $P$, Talbi $H$ Electrochemical Properties and Fourier Transform-Infrared Spectroscopic Investigations of the Redox Behaviour of Poly(Indole-5-Carboxylic Acid) in $\mathrm{LiClO}_{4}$-Acetonitrile Solutions. Spectrochimica Acta Part A: Moleculae and Biomolecular Spectroscopy 2003; 59(1): 163-168. https://doi.org/10.1016/S1386-1425(02)00150-6

[14] Khayyam H, Jazar RN, Nunn S, Golkarnarenji G, Badii K, Fakhrhoseini SM, Kumar S, Naebe M. PAN Precursor Fabrication, Applications and Thermal Stabilization Process in Carbon Fiber Production: Experimental and Mathematical Modelling. Progress in Materials Science 2020; 107: 100575 https://doi.org/10.1016/j.pmatsci.2019.100575

[15] Maynard HD, Lyu SP, Fredrickson GH, Wudl F, Chmelka BF. Syntheses of Nanophase-Segregated Poly(Vinyl Acetate)Poly(Dimethylsiloxane) and Poly(Vinyl Acetate)Poly(Styrene) Graft Copolymers. Polymer 2001; 42 (18): 7567-7574.

https://doi.org/10.1016/S0032-3861(01)00230-0

[16] Billaud D, Maarouf EB, Hannecart E. Chemical Oxidation and Polymerization of İndole. Synthetic Metals 1995; 69 (1-3): 571-572.

https://doi.org/10.1016/0379-6779(94)02573-H

[17] Blanco I, Abate L, Bottino FA, Bottino P. Thermal Degradation of Hepta Cyclopentyl, Mono Phenyl-Polyhedral Oligomeric Silsesquioxane (hcp-POSS)/Polystyrene (PS) Nanocomposites. Polymer Degradation and Stability 2012; 97 (6): 849-855. https://doi.org/10.1016/j.polymdegradstab.2012.03.041

[18] Chansai P, Sirivat A, Niamlang S, Chotpattananont D, Viravaidya-Pasuwat K. Controlled Transdermal Iontophoresis of Sulfosalicylic Acid from Polypyrrole/Poly(acrylic acid) Hydrogel. International Journal of Pharmaceutics 2009; 381(1): 25-33.

https://doi.org/10.1016/j.ijpharm.2009.07.019

[19] Urkmez G, Sarı B, Unal HI. Synthesis and Characterization of Novel Poly(dimethylsiloxane)/Polyindole Composites Journal of Applied Polymer Science 2011; 121 (3): 16001609. https://doi.org/10.1002/app.33720

[20] Aasmundtveit KE, Samuelsent EJ, Pettersson LAA, Inganas $O$, Johansson T, Feidenhans R. Structure of Thin Films of Poly(3,4-Ethylenedioxythiophene). Synthetic Metals 1999; 101(1-3): 561-564. https://doi.org/10.1016/S0379-6779(98)00315-4

[21] Bahrami A, Talib ZA, Yunus WMM, Behzad K, Abdi MM, Din FU. Low Temperature Hall Effect Investigation of Conducting Polymer-Carbon Nanotubes Composite Network. International Journal of Molecular Sciences 2012; 13 (11): 14917-14928.

https://doi.org/10.3390/ijms131114917

[22] Martens HCF, Blom PWM, Schoo HFM. Comparative Study of Hole Transport in Poly(p-Phenylene Vinylene) Derivatives. Phyical Review B 2000; 61: 7489. https://doi.org/10.1103/PhysRevB.61.7489

[23] Elashmawi IS, Hakeem NA, Abdelrazek EM. Spectroscopic And Thermal Studies of PS/PVAc Blends. Physica B: Condensed Matter 2008; 403 (19-20): 3547-3552. https://doi.org/10.1016/j.physb.2008.05.024

[24] Taylan NB, Sarı B, Unal HI. Preparation of Conducting Poly(Vinyl Chloride)/Polyindole Composites and Freestanding Films via Chemical Polymerization. Journal of Polymer Science Part B: Polymer Physics 2010; 48 (12): 1290-1298.

https://doi.org/10.1002/polb.22023 
[25] Nie G, Han X, Zhang S, Xu J, Cai T. Electrochemical Copolymerization of Indole and 3-Methylthiophene. Journal of Applied Polymer Science 2007; 104 (5): 3129-3136. https://doi.org/10.1002/app.25861

[26] Sarı B, Talu M. Electrochemical Copolymerization of Pyrrole and Aniline. Synthetic Metals 1998; 94 (2): 221-227. https://doi.org/10.1016/S0379-6779(98)00010-1

[27] Gök A, Sarı B, Talu M. Chemical Preparation of Conducting Polyfuran/Poly(2-Chloroaniline) Composites and Their Properties: A Comparison of Their Components, Polyfuran and Poly(2-Chloroaniline). Journal of Applied Polymer Science 2003; 88 (13): 2924-2931. https://doi.org/10.1002/app.12006

[28] Palaniappan S. Narayana BH. Temperature Effect on Conducting Polyaniline Salts: Thermal and Spectral Studies. Journal of Polymer Science Part A: Polymer Chemistry 1994; 32 (13): 2431-2436. https://doi.org/10.1002/pola.1994.080321304
[29] Amano K, Ishikawa H, Kobayashi A, Satoh M, Hasegawa E. Thermal Stability of Chemically Synthesized Polyaniline. Synthetic Metals 1994; 62 (3): 229-232. https://doi.org/10.1016/0379-6779(94)90210-0

[30] Tai Q, Kan Y, Chen L, Xing W, Hu Y, Song L. Morphologies and Thermal Properties of Flame-Retardant Polystyrene/aZirconium Phosphate Nanocomposites. Reactive and Functional Polymers 2010; 70 (6): 340-345 https://doi.org/10.1016/j.reactfunctpolym.2010.02.008

[31] Eşsiz S, Sarı B. Synthesis, Characterization and Hall Effect Studies of Polystyrene/Polyindole Composites. Russian Journal of Physical Chemistry B, Chemical Physics of Polymer Materials 2016; 10(4): 679-686. https://doi.org/10.1134/S1990793116040035

Received on 04-09-2020

Accepted on 07-10-2020

Published on 21-10-2020

DOI: https://doi.org/10.31875/2410-4701.2020.07.04

\section{(C) 2020 Soykan and Candoğan; Zeal Press}

This is an open access article licensed under the terms of the Creative Commons Attribution Non-Commercial License (http://creativecommons.org/licenses/by-nc/3.0/) which permits unrestricted, non-commercial use, distribution and reproduction in any medium, provided the work is properly cited. 